\title{
FROM METABOLIC NETWORKS TO MINIMAL DYNAMIC BIOREACTION MODELS
}

\author{
Agnès Provost* and Georges Bastin *,1 \\ Yves-Jacques Schneider ${ }^{* *}$ \\ * Center for Systems Engineering and Applied Mechanics \\ Université Catholique de Louvain \\ 4, Avenue G. Lemaitre, 1348 Louvain-La-Neuve, Belgium \\ ** Institut des Sciences de la Vie, \\ Université Catholique de Louvain \\ 4-5, Croix du Sud, 1348 Louvain-La-Neuve, Belgium
}

\begin{abstract}
The paper deals with the design of minimal dynamic bioreaction models in the situation where (a) the model is based on the knowledge of a detailed underlying metabolic network, (b) measurements of extra-cellular species in the reactor are the only available measurements. A brief but rigorous presentation of the theory is first given. Then the approach is illustrated with the example of chinese hamster ovary cells cultivated in stirred flasks. Copyright (c)2007 IFAC
\end{abstract}

Keywords: Bioreaction, Convex basis, Elementary flux mode, Metabolic network, Model reduction

\section{INTRODUCTION}

The issue of quantitative bioprocess modelling from extracellular measurements is a central issue in bioengineering (e.g. Nielsen et al. (2002)). In classical macroscopic models, the biomass is just viewed as a catalyst for the conversion of substrates into products. The process is represented by a set of so-called bioreactions that directly connect the substrates to the products, without making an explicit reference to the intracellular metabolism.

Recently, various publications have dealt with a new "macro-micro" approach that aims at linking the macroscopic model design to the metabolism

\footnotetext{
1 This paper presents research results of the Belgian Programme on Interuniversity Attraction Poles, initiated by the Belgian Federal Science Policy Office. The scientific responsibility rests with its author(s).
}

(e.g. Provost and Bastin (2004), Haag et al. (2005), Provost et al. (2006), Zhou et al. (2006)). Our concern in this paper is to give a brief but rigorous presentation of the theory that grounds this methodology. The goal is to design minimal dynamic bioreaction models in the situation where (a) the model is based on the knowledge of a detailed underlying metabolic network, (b) measurements of extra-cellular species in the reactor are the only available measurements. We follow a systematic "model reduction" approach that automatically produces a family of equivalent models involving a minimal set of bioreactions while being fully compatible with the underlying metabolism and consistent with the available experimental data.

Furthermore, as a matter of illustration and motivation to the theory, we consider the example of 
chinese hamster ovary $(\mathrm{CHO})$ cells cultivated in batch mode in stirred flasks (Ballez et al. (2004)).

\section{THEORY}

The intracellular metabolism of the cells under consideration is supposed to be represented by a metabolic network. A metabolic network is a directed hypergraph ${ }^{2}$ that encodes a set of elementary biochemical reactions that take place within the cell. In this hypergraph, the nodes represent the involved metabolites and the edges represent the metabolic fluxes. A typical example of metabolic network that will be considered in Section 3 is shown in Fig.1. The metabolic network involves two groups of nodes: boundary nodes and internal nodes. Boundary nodes have only either incoming or outgoing edges, but not both together. Boundary nodes can be further separated into input (or initial) and output (or terminal) nodes. Input nodes correspond to substrates that are supposed to be only consumed but not produced. Output nodes correspond to final products that are supposed to be only produced but not consumed. In contrast, the internal (or intermediary) nodes are the nodes that have necessarily both incoming and outgoing incident edges. They correspond to metabolites that are produced by some of the metabolic reactions and consumed by other reactions inside the cell.

It is assumed that the cells are cultivated in batch mode in a stirred tank reactor. The dynamics of substrates and products in the bioreactor are represented by the following basic differential equations:

$$
\begin{aligned}
\frac{d \mathbf{s}(t)}{d t} & =-\mathbf{v}_{s}(t) X(t) \\
\frac{d \mathbf{p}(t)}{d t} & =\mathbf{v}_{p}(t) X(t)
\end{aligned}
$$

where $X(t)$ is the biomass concentration in the culture medium, $\mathbf{s}(t)$ is the vector of substrate concentrations, $\mathbf{p}(t)$ the vector of product concentrations, $\mathbf{v}_{s}(t)$ the vector of specific uptake rates and $\mathbf{v}_{p}(t)$ the vector of specific production rates. (From now on, the time index " $t$ " will be omitted).

Obviously, the specific rates $\mathbf{v}_{s}$ and $\mathbf{v}_{p}$ are not independent. They are quantitatively related through the intracellular metabolism represented by the metabolic network. In order to explicit this relation, the quasi steady-state paradigm of metabolic flux analysis (MFA) is adopted (e.g. Stephanopoulos et al. (1998)). This means that for each internal metabolite of the network, it is assumed that the net sum of production and

\footnotetext{
2 A hypergraph is a generalization of a graph, where edges can connect any number of vertices.
}

consumption fluxes, weighted by their stoichiometric coefficients, is zero. This is expressed by the algebraic relation:

$$
\mathbf{N v}=0 \quad \mathbf{v} \geqslant 0
$$

where $\mathbf{v}=\left(v_{1}, v_{2}, \ldots, v_{m}\right)^{T}$ is the m-dimensional vector of fluxes and $\mathbf{N}=\left[n_{i j}\right]$ is the $n \times m$ stoichiometric matrix of the metabolic network ( $m$ is the number of fluxes and $n$ the number of internal nodes of the network). More precisely, a flux $v_{j}$ denotes the rate of reaction $j$ and a non-zero $n_{i j}$ is the stoichiometric coefficient of metabolite $i$ in reaction $j$.

For a given metabolic network, the set $\mathcal{S}$ of admissible flux distributions is the set of vectors $\mathbf{v}$ that satisfy the finite set (2) of homogeneous linear equalities and inequalities. Each admissible $\mathbf{v}$ must necessarily be non-negative and belong to the kernel of the matrix $\mathbf{N}$. Hence the set $\mathcal{S}$ is the pointed polyhedral cone which is the intersection of the kernel of $\mathbf{N}$ and the nonnegative orthant. This implies that any flux distribution $\mathbf{v}$ can be expressed as a non-negative linear combination of a set of vectors $\mathbf{e}_{i}$ which are the edges (or extreme rays) of the polyhedral cone and form therefore a unique convex basis (see e.g. Weyl (1950)) of the flux space:

$$
\mathbf{v}=w_{1} \mathbf{e}_{1}+w_{2} \mathbf{e}_{2}+\cdots+w_{p} \mathbf{e}_{p} \quad w_{i} \geqslant 0 .
$$

The $m \times p$ non-negative matrix $\mathbf{E}$ with column vectors $\mathbf{e}_{i}$ obviously satisfies $\mathbf{N E}=0$ and (3) is written in matrix form as

$$
\mathbf{v}=\mathbf{E w} \quad \text { with } \quad \mathbf{w} \triangleq\left(w_{1}, w_{2}, \ldots, w_{p}\right)^{T} .
$$

From a metabolic viewpoint, the vectors $\mathbf{e}_{i}$ of the convex basis encode the simplest metabolic paths that connect the substrates (input nodes) to the products (output nodes). More precisely, the non-zero entries of a basis vector $\mathbf{e}_{i}$ enumerate the fluxes of a sequence of biochemical reactions starting at one or several substrates and ending at one or several products. These simple pathways between substrates and products are called extreme pathways (ExPa) or elementary flux modes (EFM) of the network (Schuster et al. (1999) and Nielsen et al. (2002)). Since the intermediary reactions are assumed to be at quasi steady-state, a single macroscopic bioreaction is then readily defined from an elementary flux mode by considering only the involved initial substrates and final products.

Let us now come back to the basic model (1) in order to elucidate the relation between the specific consumption and production rates $\mathbf{v}_{s}$ and $\mathbf{v}_{p}$ induced by the metabolic network. Obviously $\mathbf{v}_{s}$ and $\mathbf{v}_{p}$ are linear combinations of some of the metabolic fluxes. This is expressed by defining appropriate matrices $\mathbf{N}_{s}$ and $\mathbf{N}_{p}$ such that

$$
\mathbf{v}_{s}=\mathbf{N}_{s} \mathbf{v} \quad \mathbf{v}_{p}=\mathbf{N}_{p} \mathbf{v} .
$$


From (4) and (5), it follows that the model (1) is rewritten as:

$$
\frac{d}{d t}\left(\begin{array}{c}
\mathbf{s} \\
\mathbf{p}
\end{array}\right)=\left(\begin{array}{c}
-\mathbf{N}_{s} \\
\mathbf{N}_{p}
\end{array}\right) \mathbf{E w} X=\mathbf{K}_{\mathbf{e}} \mathbf{w} X
$$

where

$$
\mathbf{K}_{\mathbf{e}} \triangleq\left(\begin{array}{c}
-\mathbf{N}_{s} \\
\mathbf{N}_{p}
\end{array}\right) \mathbf{E}
$$

is the stoichiometric matrix of the set of bioreactions encoded by the EFMs. Equation (6) can be regarded as the dynamic model of a bioprocess governed by the bioreactions with stoichiometry $\mathbf{K}_{\mathbf{e}}$ and specific reaction rates $\mathbf{w}$. In other terms, each weighting coefficient $w_{i}$ in (3) can equally be interpreted as the specific reaction rate of the bioreaction encoded by the EFM $\mathbf{e}_{i}$ : the flux vector $\mathbf{v}$ is thus a linear combination of EFMs whose non-negative weights are the macroscopic bioreaction rates $w_{i}$.

However an important issue concerns the number of distinct bioreactions that are generated when computing the EFMs. It may become very large because it combinatorially increases with the size of the underlying metabolic network ${ }^{3}$. Furthermore, even when the number of EFMs is rather limited, it appears that the resulting set of bioreactions can be significantly redundant for the design of a dynamic model that fully explains the available experimental data. There is therefore clearly a need for reducing the model size as much as possible and trying to determine a minimal subset of bioreactions that are able to fully describe the available experimental data.

As mentionned above, our concern is dynamic modelling when measurements of extracellular species in the culture medium are available. We assume that the specific uptake and excretion rates $v_{s i}$ and $v_{p i}$ of those measured extracellular species are estimated from the data and collected in a vector $\mathbf{v}_{m}$ such that:

$$
\mathbf{N}_{\mathbf{m}} \mathbf{v}=\mathbf{v}_{m}
$$

for some appropriate matrix

$$
\mathbf{N}_{\mathbf{m}} \text { sub-matrix of }\left(\begin{array}{l}
\mathbf{N}_{s} \\
\mathbf{N}_{p}
\end{array}\right) \text {. }
$$

In order to be compatible with the experimental measurements, an admissible flux distribution $\mathbf{v}$ has now to satisfy the augmented system:

\footnotetext{
3 The Double Description (DD) method (Motzkin et al. (1953)) is the simplest known algorithm for computing the convex basis of the solution space (see Fukuda and Prodon (1996) for a review). In the context of metabolic networks various refinements have been proposed that differ from the original DD algorithm mainly by their initialization. A first specific algorithm was presented by Schuster and Schuster (1993). Recently, the implementation of the DD method for metabolic networks has received various further improvements (e.g. Gagneur and Klamt (2004) and Klamt et al. (2005)).
}

$$
\left(\begin{array}{c}
\mathbf{N} \\
\mathbf{N}_{\mathbf{m}}
\end{array}\right) \mathbf{v}=\left(\begin{array}{c}
\mathbf{0} \\
\mathbf{v}_{m}
\end{array}\right)
$$

Here we focus on the special case where this system is exactly determined ${ }^{4}$ and has a single well-defined solution which can obviously be decomposed in the convex basis as expressed by (3). But even if the flux vector $\mathbf{v}$ satisfying equation (9) is unique, it must be emphasized that the decomposition of $\mathbf{v}$ in the convex basis $\left\{\mathbf{e}_{i}\right\}$ is not unique which is the algebraic expression of the fact that the set of bioreactions used in the dynamical model (6) is redundant. Using (4), system (9) is equivalent to the system:

$$
\left(\begin{array}{c}
\mathbf{N E} \\
\mathbf{N}_{\mathbf{m}} \mathbf{E}
\end{array}\right) \mathbf{w}=\left(\begin{array}{c}
\mathbf{0} \\
\mathbf{v}_{m}
\end{array}\right) \quad \mathbf{w} \geqslant 0 .
$$

We observe that the first equation $\mathbf{N E w}=\mathbf{0}$ is trivially satisfied independently of $\mathbf{w}$ since $\mathbf{N E}=$ 0 by definition. Hence, system (10) may be reduced to the second equation:

$$
\mathbf{N}_{\mathbf{m}} \mathbf{E w}=\mathbf{v}_{m} \quad \mathbf{w} \geqslant 0 .
$$

or equivalently:

$$
\left(\mathbf{N}_{\mathbf{m}} \mathbf{E}-\mathbf{v}_{m}\right)\left(\begin{array}{c}
\mathbf{w} \\
1
\end{array}\right)=0 \quad \mathbf{w} \geqslant 0 .
$$

In this form, it is clear that the set of admissible reaction rate vectors $\mathbf{w}$ that satisfy (11) also constitutes a convex polyhedral cone. Therefore there exists a set of appropriate edge vectors $\mathbf{h}_{i}$ such that any arbitrary convex combination of the form:

$$
\mathbf{w}=\sum_{i} z_{i} \mathbf{h}_{i} \quad z_{i} \geqslant 0 \quad \sum_{i} z_{i}=1
$$

is necessarily an admissible $\mathbf{w}$ satisfying (11). The convex basis vectors $\mathbf{h}_{i}$ have an important and critical property : the number of non-zero entries is at most equal to the number of measured uptake and excretion rate i.e. the size of the vector $\mathbf{v}_{\mathbf{m}}$ (see Fukuda and Prodon (1996) and Section 3.5 in Provost (2006)). From a metabolic viewpoint, each vector $\mathbf{h}_{i}$ is a particular solution $\mathbf{w}$ of (11), or equivalently a particular way (among an infinity) of computing the flux distribution $\mathbf{v}$ that satisfies (10):

$$
\mathbf{v}=\mathbf{E h}_{i} \quad \forall i
$$

Of course in this expression, the non-zero entries of the vector $\mathbf{h}_{i}$ are interpreted as the weights of the respective contributions of the corresponding EFMs in the computation of the flux distribution $\mathbf{v}$. But, at the same time, they can also be interpreted as being the specific rates of the bioreactions that are encoded by the EFMs and are involved in the dynamic model (6).

Hence each convex basis vector $\mathbf{h}_{i}$ brings two different pieces of information. First it tells which

\footnotetext{
4 The case where system (9) is underdetermined is treated in Provost et al. (2006) and Provost (2006).
} 
EFMs and consequently which bioreactions are sufficient to explain the measured uptake and excretion rates $\mathbf{v}_{\mathbf{m}}$. These EFMs are designated by the position of the non-zero entries of $\mathbf{h}_{\mathbf{i}}$. Secondly, the value of each non-zero entry of $\mathbf{h}_{i}$ is the value of the reaction rate of the corresponding bioreaction.

For each basis vector $\mathbf{h}_{i}$, we can then define a selection matrix $\mathbf{S}_{\mathbf{i}}$ that encodes the corresponding selection of bioreactions. Then the dynamical model (6) is reduced to a minimal form:

$$
\frac{d}{d t}\left(\begin{array}{l}
\mathbf{s} \\
\mathbf{p}
\end{array}\right)=\mathbf{K}_{\mathbf{i}} \mathbf{r}_{i} X
$$

where $\mathbf{K}_{\mathbf{i}} \triangleq \mathbf{K}_{\mathbf{e}} \mathbf{S}_{\mathbf{i}}$ and $\mathbf{r}_{i} \triangleq\left(\mathbf{S}_{\mathbf{i}}\right)^{T} \mathbf{h}_{i}$ respectively denote the stoichiometric matrix and the vector of the specific reaction rates of the selected minimal set of bioreactions.

Therefore, we see that the computation of the convex basis vectors $\mathbf{h}_{i}$ provides the tool for determining all the minimal dynamical models that are both compatible with the metabolic network and the available measurements. Furthermore, it is worth to clearly understand that all of these minimal models are totally equivalent because they all provide exactly the same internal flux distribution and the same dynamical simulation results.

In the next section we shall illustrate this methodology with an application to chinese hamster ovary $(\mathrm{CHO})$ cells cultivated in batch mode in stirred flasks.

\section{CASE-STUDIES}

Two case-studies will be given in the oral presentation of this paper. The first one is a simple example that involves only two substrates (glucose and glutamine) and a rather limited metabolic network with only nine EFMs. It allows to illustrate clearly that even with a small number of EFMs, the resulting set of bioreactions can be significantly redundant. The second example will involve all the amino-acids as substrates and a much more complex metabolic network with more than 80000 EFMs which obviously makes model reduction imperative. Within the size limits of the present paper, only the first example is briefly described. The second example can be found in Provost (2006).

During the growth phase, the cell metabolism is described by the metabolic network presented in Fig.1. This network describes only the part of the metabolism concerned with the utilisation of the two main energetic nutrients (glucose and glutamine). The metabolism of the amino acids provided by the culture medium is not considered
Table 1. Matrix $\mathbf{E}$ of elem. flux modes.

\begin{tabular}{cccccccccc}
\hline & $\mathbf{e}_{\mathbf{1}}$ & $\mathbf{e}_{\mathbf{2}}$ & $\mathbf{e}_{\mathbf{3}}$ & $\mathbf{e}_{\mathbf{4}}$ & $\mathbf{e}_{\mathbf{5}}$ & $\mathbf{e}_{6}$ & $\mathbf{e}_{\mathbf{7}}$ & $\mathbf{e}_{\mathbf{8}}$ & $\mathbf{e}_{\mathbf{9}}$ \\
\hline$v_{1}$ & 1 & 1 & 0 & 0 & 0 & 2 & 2 & 2 & 2 \\
$v_{2}$ & 1 & 1 & 0 & 0 & 0 & 0 & 0 & 0 & 0 \\
$v_{3}$ & 0 & 0 & 0 & 0 & 0 & 2 & 2 & 2 & 2 \\
$v_{4}$ & 1 & 1 & 0 & 0 & 0 & 0 & 0 & 0 & 0 \\
$v_{5}$ & 2 & 2 & 0 & 0 & 0 & 0 & 0 & 0 & 0 \\
$v_{6}$ & 2 & 0 & 0 & 1 & 0 & 2 & 1 & 0 & 0 \\
$v_{7}$ & 0 & 0 & 1 & 0 & 0 & 0 & 1 & 1 & 0 \\
$v_{8}$ & 0 & 2 & 0 & 0 & 1 & 0 & 0 & 1 & 2 \\
$v_{9}$ & 0 & 2 & 0 & 0 & 1 & 0 & 0 & 1 & 2 \\
$v_{10}$ & 0 & 2 & 0 & 0 & 1 & 0 & 0 & 1 & 2 \\
$v_{11}$ & 0 & 2 & 1 & 1 & 2 & 3 & 3 & 4 & 5 \\
$v_{12}$ & 0 & 2 & 1 & 1 & 2 & 4 & 4 & 5 & 6 \\
$v_{13}$ & 0 & 2 & 0 & 0 & 1 & 2 & 2 & 3 & 4 \\
$v_{14}$ & 0 & 0 & 1 & 1 & 1 & 2 & 2 & 2 & 2 \\
$v_{15}$ & 0 & 0 & 0 & 0 & 0 & 2 & 2 & 2 & 2 \\
$v_{16}$ & 0 & 0 & 0 & 1 & 1 & 1 & 0 & 0 & 1 \\
$v_{17}$ & 0 & 0 & 1 & 1 & 1 & 0 & 0 & 0 & 0 \\
$v_{18}$ & 0 & 0 & 0 & 0 & 0 & 1 & 1 & 1 & 1 \\
$v_{19}$ & 0 & 6 & 2 & 2 & 5 & 6 & 6 & 9 & 12 \\
\hline
\end{tabular}

and the pentose-phosphate pathway is neglected. Moreover it is assumed that a part of the glutamine is used for the making of nucleotides which are lumped into a single species with equal shares of purines and pyrimidines (see Provost et al. (2006) and Provost (2006) for further motivation and details).

In this network, there are

- two input substrates : Glucose (Glc) and Glutamine (Gln) ;

- five output products : Lactate (Lac), $\mathrm{CO}_{2}$, $\mathrm{NH}_{4}$, Alanine (Ala) and Nucleotides (Nucl) ;

- $n=14$ internal metabolites : Glucose6-phosphate, Dihydroxy-acetone-phosphate, Ribose-5-phosphate, Glyceraldehyde-3 phosphate, Pyruvate, Acetyl-coA, Citrate, $\alpha$ ketoglutarate, Fumarate, Malate, Oxaloacetate, Aspartate, Glutamate, $\mathrm{CO}_{2}$;

- $m=19$ metabolic fluxes denoted $v_{1}$ to $v_{19}$ in Fig. 1.

Without loss of generality, all the intermediate metabolites that are not located at branch points are omitted from the network. The network has nine EFMs ${ }^{5}$ that are collected in matrix $\mathbf{E}$ (see Table 1) and from which the following set of input/output bioreactions is readily derived:

(b1) Glc $\rightarrow 2$ Lac

(b2) $\mathrm{Glc} \rightarrow 6 \mathrm{CO}_{2}$

(b3) $\mathrm{Gln} \rightarrow \mathrm{Ala}+\mathrm{NH}_{4}+2 \mathrm{CO}_{2}$

(b4) $\mathrm{Gln} \rightarrow \mathrm{Lac}+2 \mathrm{NH}_{4}+2 \mathrm{CO}_{2}$

(b5) Gln $\rightarrow 2 \mathrm{NH}_{4}+5 \mathrm{CO}_{2}$

(b6) $2 \mathrm{Glc}+\mathrm{Gln} \rightarrow 2 \mathrm{Lac}+\mathrm{Nucl}+\mathrm{NH}_{4}+6 \mathrm{CO}_{2}$

(b7) $2 \mathrm{Glc}+\mathrm{Gln} \rightarrow \mathrm{Lac}+\mathrm{Ala}+\mathrm{Nucl}+6 \mathrm{CO}_{2}$

(b8) $2 \mathrm{Glc}+\mathrm{Gln} \rightarrow \mathrm{Ala}+\mathrm{Nucl}+9 \mathrm{CO}_{2}$

(b9) $2 \mathrm{Glc}+\mathrm{Gln} \rightarrow \mathrm{Nucl}+\mathrm{NH}_{4}+12 \mathrm{CO}_{2}$

Moreover, there are five measured extra-cellular species : the two substrates (Glucose and Glu-

\footnotetext{
5 computed with METATOOL (Pfeiffer et al. (1999))
} 


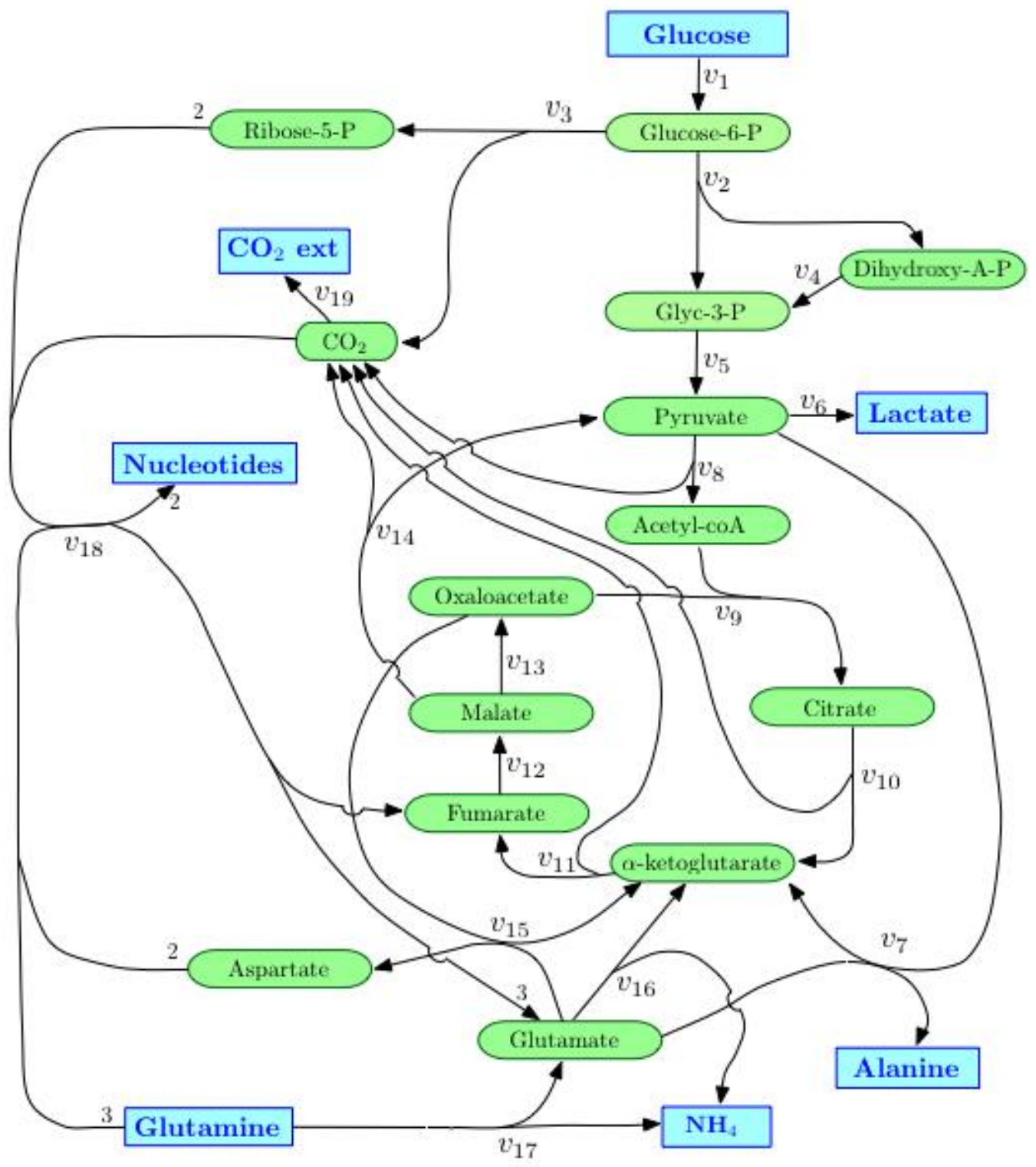

Fig. 1. Metabolic network : rectangular boxes represent input/output nodes, elliptic boxes represent internal nodes. (The numbers along some arrows indicate stoichiometric coefficients).

tamine) and three excreted products (Lactate, Ammonia, Alanine). The values of the average specific uptake and excretion rates (vector $\mathbf{v}_{\mathbf{m}}$ ), computed by linear regression during the growth phase (see Provost and Bastin (2004)), are given in Table 2

Table 2. Specific uptake and excretion rates $\left(\mathrm{mM} /\left(\mathrm{d} \times 10^{9}\right.\right.$ cells $\left.)\right)$.

\begin{tabular}{ccccc}
\hline Glucose & Glutamine & Lactate & $\mathrm{NH}_{4}$ & Alanine \\
\hline 4.0546 & 1.1860 & 7.3949 & 0.9617 & 0.2686 \\
\hline
\end{tabular}

In this application, it can be checked that system (9) is a system of 19 equations with 19 unknowns which is uniquely determined. We are then in a position to compute the set of vectors $\mathbf{h}_{i}$ and the result is shown in Table 4 . We first observe that there are 12 different vectors $\mathbf{h}_{\mathbf{i}}$ in this Table. They all produce exactly the same value of the flux distribution $\mathbf{v}$ when premultiplied by the matrix $\mathbf{E}$ as expected according to (13) (see Table 3). Furthermore, as predicted by the

Table 3. Metabolic fluxes.

\begin{tabular}{ccccc}
\hline$v_{1}$ & $v_{2}$ & $v_{3}$ & $v_{4}$ & $v_{5}$ \\
4.0546 & 3.5979 & 0.4567 & 3.5979 & 7.1958 \\
\hline$v_{6}$ & $v_{7}$ & $v_{8}$ & $v_{9}$ & $v_{10}$ \\
7.3949 & 0.2686 & 0.4900 & 0.4900 & 0.4900 \\
\hline$v_{11}$ & $v_{12}$ & $v_{13}$ & $v_{14}$ & $v_{15}$ \\
1.6760 & 1.9043 & 0.9467 & 0.9577 & 0.4567 \\
\hline$v_{16}$ & $v_{17}$ & $v_{18}$ & $v_{19}$ & \\
0.4607 & 0.5010 & 0.2283 & 3.8420 & \\
\hline
\end{tabular}

theory, we also observe that there are exactly 5 non-zero entries in each vector $\mathbf{h}_{\mathbf{i}}$. From these observations, we can conclude that there are 12 different equivalent minimal dynamical models of the form (14) for the considered process. For each of these models, Table 4 tells us which 5 
Table 4. Specific reaction rates for the 12 equivalent minimal dynamic models $\left(\mathrm{mM} /\left(\mathrm{d} \times 10^{9}\right.\right.$ cells $)$

\begin{tabular}{ccccccccccccc}
\hline & $\mathbf{h}_{\mathbf{1}}$ & $\mathbf{h}_{\mathbf{2}}$ & $\mathbf{h}_{\mathbf{3}}$ & $\mathbf{h}_{\mathbf{4}}$ & $\mathbf{h}_{\mathbf{5}}$ & $\mathbf{h}_{\mathbf{6}}$ & $\mathbf{h}_{\mathbf{7}}$ & $\mathbf{h}_{\mathbf{8}}$ & $\mathbf{h}_{\mathbf{9}}$ & $\mathbf{h}_{\mathbf{1 0}}$ & $\mathbf{h}_{\mathbf{1 1}}$ & $\mathbf{h}_{\mathbf{1 2}}$ \\
\hline (b1) & 3.5833 & 3.4671 & 3.3529 & 3.5979 & 3.4691 & 3.5979 & 3.5979 & 3.5979 & 3.3529 & 3.5979 & 3.5813 & 3.5979 \\
(b2) & 0.0146 & 0.1308 & 0.2450 & 0.0 & 0.1288 & 0.0 & 0.0 & 0.0 & 0.2450 & 0.0 & 0.0167 & 0.0 \\
(b3) & 0.0403 & 0.0403 & 0.0403 & 0.0403 & 0.2686 & 0.2686 & 0.1398 & 0.0403 & 0.2686 & 0.0695 & 0.2686 & 0.2686 \\
(b4) & 0.0 & 0.4607 & 0.4607 & 0.0 & 0.0 & 0.0 & 0.0 & 0.1991 & 0.2324 & 0.0 & 0.2324 & 0.1991 \\
(b5) & 0.4607 & 0.0 & 0.0 & 0.4607 & 0.2324 & 0.2324 & 0.3612 & 0.2617 & 0.0 & 0.4314 & 0.0 & 0.0333 \\
(b6) & 0.0 & 0.0 & 0.0 & 0.0 & 0.2283 & 0.0995 & 0.0995 & 0.0 & 0.2283 & 0.0 & 0.0 & 0.0 \\
(b7) & 0.2283 & 0.0 & 0.2283 & 0.1991 & 0.0 & 0.0 & 0.0 & 0.0 & 0.0 & 0.1991 & 0.0 & 0.0 \\
(b8) & 0.0 & 0.2283 & 0.0 & 0.0293 & 0.0 & 0.0 & 0.1288 & 0.2283 & 0.0 & 0.0 & 0.0 & 0.0 \\
(b9) & 0.0 & 0.0 & 0.0 & 0.0 & 0.0 & 0.1288 & 0.0 & 0.0 & 0.0 & 0.0293 & 0.2283 & 0.2283 \\
\hline
\end{tabular}

bioreactions (among the set (b1)-(b9)) are used and the value of their reaction rates.

The design of a particular dynamic bioreaction model is finally completed by chosing arbitrarily any vector $\mathbf{h}_{\mathbf{i}}$ in Table 4 and assuming that the selected bioreactions have Michaelis-Menten kinetics with maximum specific rates $\mu_{i}$ given by the non-zero entries of $\mathbf{h}_{\mathbf{i}}$. This automatically gives by construction a model which necessarily produces simulations that fit the experimental data with a high accuracy, as it is illustrated by many examples in Provost and Bastin (2004), Provost et al. (2006) and Provost (2006).

\section{REFERENCES}

J.S. Ballez, J. Mols, J. Burteau, S.N. Agathos, and Y-J. Schneider. Plant protein hydrolysates support cho-320 cells proliferation and recombinant ifn-gamma production in suspension and inside microcarriers in protein-free media. $C y$ totechnology, 44:103-114, 2004.

K. Fukuda and A. Prodon. The double description method revisited. In R. Euler and M. E. Deza I. Manoussakis, editors, Combinatorics and Computer Science, volume 1120 of Lecture Notes in Computation Sciences, pages 91-111. Springer-Verlag, 1996.

J. Gagneur and S. Klamt. Computation of elementary modes : a unifying framework and the new binary approach. BMC Bioinformatics, 5: 175, 2004.

J.E. Haag, A. Vande Wouwer, and P. Bogaerts. Dynamic modeling of complex biological systems : a link between metabolic and macroscopic descriptions. Mathematical Biosciences, 193:25-49, 2005.

S. Klamt, J. Gagneur, and A. von Kamp. Algorithmic approaches for computing elementary modes in large biochemical networks. IEE Proceedings Systems Biology, 152:249-255, 2005.

T.S. Motzkin, H. Raiffa, G.L. Thompson, and R.M. Thrall. The double description method. In H.W. Kuhn and A.W. Tucker, editors, Contribution to the Theory of Games Vol. II, volume 28 of Annals of Mathematical Stud- ies, pages 51-73, Princeton, New Jersey, 1953. Princeton University Press.

J. Nielsen, J. Villadsen, and G. Liden. Bioreaction Engineering Principles. Kluwer Academic, 2002.

T. Pfeiffer, I. Sanchez-Valdenebro, J.C. Nu, F. Montero, and S. Schuster. Metatool : for studying metabolic networks. Bioinformatics, 15(3):251-257, March 1999.

A. Provost. Metabolic design of dynamic bioreaction models. PhD thesis, Faculty of Engineering, Université Catholique de Louvain, November 2006.

A. Provost and G. Bastin. Dynamical metabolic modelling under the balanced growth condition. Journal of Process Control, 14(7):717-728, 2004.

A. Provost, G. Bastin, S.N. Agathos, and Y-J. Schneider. Metabolic design of macroscopic bioreaction models : Application to Chinese hamster ovary cells. Bioprocess and Biosystems Engineering, 2006.

R. Schuster and S. Schuster. Refined algoritm and computer program for calculating all nonnegative fluxes admissible in steady states of biochemical reaction systems with or without some flux rates fixed. Computer Applications in the Biosciences, 9(1):79-85, February 1993.

S. Schuster, D.A. Fell, and T. Dandekar. Detection of elementary flux modes in biochemical networks : a promising tool for pathway analysis and metabolic engineering. Trends in Biotechnology, 17(2):53-60, February 1999.

G. Stephanopoulos, J. Nielsen, and A. Aristidou. Metabolic Engineering : Principles and Methodologies. Academic Press, San Diego, 1998.

H. Weyl. The elementary theory of convex polyhedra. In Contributions to the Theory of Games Vol. I, Annals of Mathematical Studies, pages 3-18, Princeton, New Jersey, 1950. Princeton University Press.

F. Zhou, J-X. Bi, A-P. Zeng, and J-Q. Yuan. A macrokinetic and regulator model for myeloma cell culture based on metabolic balance of pathways. Process Biochemistry, 41:2207-2217, 2006. 